Cite as:

Desolda G. and Jetter H.C. (2015). Spatial Awareness in Mobile Devices to Compose

Data Source: A Utilization Study. In End-User Development. Díaz P., Pipek V., Ardito

C., Jensen C., Aedo I., andBoden A. (ed.), Vol. 9083, pp. 291-4. Springer International Publishing. DOI 10.1007/978-3-319-18425-8_32. Print ISBN 978-3-319-18424-1.

\title{
Spatial Awareness in Mobile Devices to Compose Data Source: a Utilization Study
}

\author{
Giuseppe Desolda ${ }^{1}$, Hans-Christian Jetter ${ }^{2}$ \\ ${ }^{1}$ Dipartimento di Informatica, Università degli Studi di Bari Aldo Moro \\ Via Orabona, 4-70125 - Bari, Italy \\ giuseppe.desolda@uniba.it \\ ${ }^{2}$ University of Applied Sciences Upper Austria, Campus Hagenberg \\ Softwarepark 11, 4232 Linz, Austria \\ hans-christian.jetter@fh-hagenberg.at
}

\begin{abstract}
The growing amount of low cost mobile devices over the last years allowed almost each person to be equipped with their personal device. This situation is leading researchers to investigate new possibilities to exploit the physical presence of several mobile devices for purposes like co-located collaborative activities. For example, today it is a common situation that a group of users tries to satisfy situational needs by using their mobile devices without the possibility to 'integrate' or join them to make more use of their full potential. In this paper, we propose a study to evaluate a new paradigm designed to compose data sources available on each mobile device through their spatial arrangement on a desk. HuddleLamp is a device that allows each mobile device to be spatially-aware of all other mobile devices on the desk. The main goal of this study is to understand if spatial awareness features can support co-located collaborative tasks to satisfy situational informational needs.
\end{abstract}

Keywords: Multi-device environments, spatially-aware interfaces, user study.

\section{Introduction}

In the last 30 years, the technological progress has encouraged the proliferation of different types of mobile devices. Their use has substantially changed over the time. Initially designed to call or send text messages to other people, they are now used primarily to visit web pages, chat, share content, pay an item, take a picture or record a video, listen music, etc. Despite the enormous advances in terms of functionalities offered by these mobile devices, until now little attention has been dedicated to the possibility of providing new opportunities by physically combining devices located in the same environment. In fact, we can now safely assume that all people in a co-located group carry a personal device, but this is rarely supported in the ways we can use multiple devices together. For example, while users are discussing a certain topic, they might want to find some information on the Web using their devices; in this case, they typically use

adfa, p. 1, 2011.

(C) Springer-Verlag Berlin Heidelberg 2014 
their devices individually or, at best, by communicating by means of apps that are usually not designed to support information sharing in a group of co-located people. To better understand this problem, let us consider the following scenario.

Alice, Rudin and Bob are three friends that have decided to move to London for studying. They meet at Alice's home to discuss renting a shared apartment. First, Alice opens on her smartphone the site Zoopla (one of the most important property rental sites in the UK) and sets some parameters like price range, number of bedrooms, property type. Then she writes 'London' in the search box and the site shows a list of about 500 results ordered by price. Rudin says that he prefers a property near a bus or metro station, thus he opens on his smartphone a UK site to retrieve information about public transportation in London. A list of transport stations is shown on his device. Furthermore, Bob opens on his smartphone a site with information about air pollution in UK to retrieve a list of air pollution stations in London. The discussion continues on the basis of property parameters, air quality and availability of public transportation. Every time they decide to consider a different location (e.g. by refining the query with a specific area of London), they have to manually refine the query on each device. At a certain point, Alice takes her tablet and opens Google Maps because she prefers to visualize the results of the smartphones on a map. From now on, each time they want to know the location of a point of interest (house, transport station, pollution air station) on the map, Alice has to query Google Maps by typing in the specific address.

In this scenario, the users are not adequately supported by ad-hoc mobile device mechanisms to perform their tasks because the devices act in isolation, thus information and queries must be manually synchronized across device boundaries by their users. Today these types of informational needs that involve different data sources are typically supported by mashup platforms that allow end users to compose their own web application on a desktop PC also providing remote collaboration mechanisms [1]. Nonetheless, these existing solutions are not intended for supporting a collaborative colocated scenario with mobile devices.

We have designed a novel paradigm that allows people to physically combine their devices and their data based on a recent technology called HuddleLamp. It is a desk lamp with an integrated depth and RGB camera that allows users to compose their mobile devices in an ad hoc fashion, just by putting them under this desk lamp and without instrumenting them with custom-built sensing hardware or markers [2]. By using HuddleLamp, each device under the lamp is tracked in space and also becomes aware of the locations of all other mobile devices on the desk. This enables our newly designed composition paradigm that allows groups of users to combine the data sources from each device (e.g., different web sites) by means of their spatial position on the HuddleLamp desk. Users are enabled to formulate their query and reconfigure the flow of data between devices by simply rearranging them in the space, ideally achieving a new kind of EUD that feels "natural": rather than feeling like "development", this paradigm resembles natural arranging of devices in space, similar to how we constantly arrange non-digital objects like sheets or piles of paper, books, or folders on our desks or conference tables. According to [3], being manipulations part of a physical and spatial "mother tongue" that we all share, such a paradigm should facilitate the composition activities performed by the users independently by their culture and context. 
We have elicited the spatial interactions for this paradigm in 2 pilot studies from 8 end users. Then we conducted 5 focus groups with 26 end users. Details about these studies are out of the scope of this paper and will be subject of future publications. However, in brief, three types of device compositions were elicited in these studies: 1) how to query multiple devices with same keyword(s) by performing the query only on one of them? 2) Given two devices A and B, how to query B with portions of text displayed on A? 3) Given the device A that shows data with a certain visualization (e.g. list of items), how we can move these data into the device B that shows input data with another type of visualization (e.g. map)? The results of the elicitation study in terms of the composition paradigm are summarized in Fig. 1.

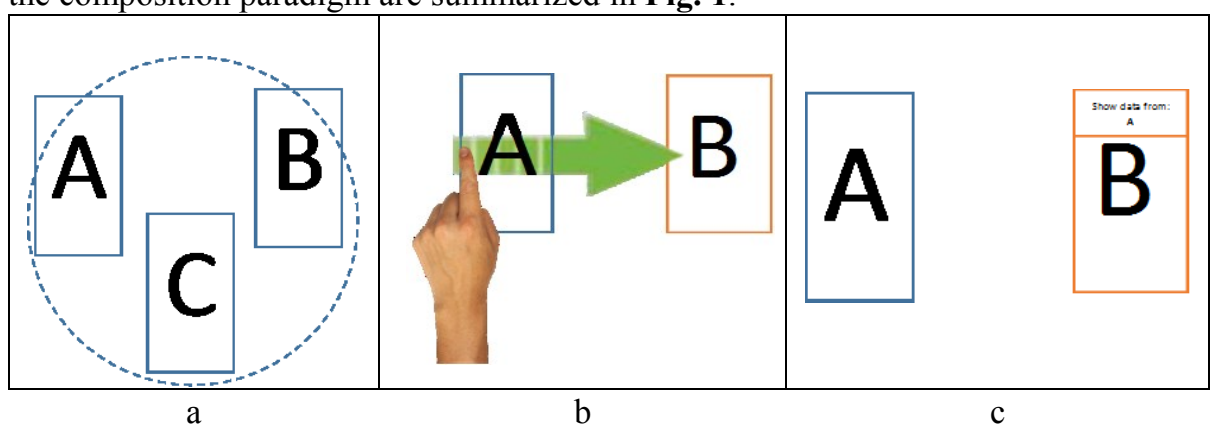

Fig. 1. a) A group of devices physically close are queried with same keyword(s) executed on one of them; b) a swipe gesture from a portion of text in the device A towards the device B causes a query on B with the swiped text; c) a menu on the device B allows to choose the device(s) A on the desk in order to visualize the results of A on B in different way.

The main goal of the hands-on activity is to understand if the spatial awareness functionality provided by HuddleLamp supports the co-located collaborative tasks performed by the end users participating in the activity. In particular, we address the following research questions: RQ1: Are the users able to perform real co-located collaborative tasks by exploiting spatial awareness mechanisms? RQ2: Do the users like to have spatial awareness mechanisms to perform co-located collaborative tasks? RQ3: Do the users prefer to exploit spatial awareness mechanisms in other ways?

In order to answer to these questions, we designed a utilization study described in the following section.

\section{Hands-on Activity: a Utilization Study}

During the days before the playground session, to motivate and recruit passers-by in participating, we will install HuddeLamp to allow anyone to interact with some demo application. We will recruit 16 people that will be divided in groups of four people. Each group will be scheduled in one-hour slots during the playground session. For each group, we will start with a quick introduction about the HuddleLamp technology. Some demo applications will be shown to explain the spatially-aware interaction. Afterwards, we will briefly introduce the three composition techniques by showing them on the 
HuddleLamp desk. Then participants will be asked to perform two tasks with a balanced complexity, so that they can be accomplished with or without spatial awareness mechanisms. Concretely, in the first task they will be asked to find a cheap property in a specific zone of London. The property has to be very close to a metro station and in a non-polluted zone (i.e., pollution value below a threshold). With this task, we try to answer our research questions w.r.t. the composition mechanisms in Fig. 1a and Fig. 1c. With the second task, each group has to choose an upcoming musical concert at their favourite location. They will be asked to use a specific web site and, if the information provided by that site are not enough to choose the concert, they should use other sites to gather further information (e.g. YouTube, Wikipedia, and Google Maps). With this task, we try to answer to our research questions w.r.t. composition mechanism in Figure $1 \mathrm{~b}$. During the interaction they will be asked to verbalize their thoughts and comment on their actions according to the think-aloud protocol.

For the duration of this study, at least one organizer will assist the users. Furthermore, all the interactions will be audio-video recorded, obviously after all participants agreed to this and a consent form was signed. After the two tasks, each group will be asked to discuss the pro and cons of the composition mechanisms and their discussion will be guided by an organizer towards the research questions. At the end, group participants will be requested to complete an online questionnaire. Each group session will be 1 hour long.

The planned study aims at investigating the proposed spatially-aware composition paradigm by involving real end users and EUD experts. On the one hand, interaction of real end users with our system could give us important feedback about understating and acceptance of the proposed paradigm. On the other hand, EUD experts will provide important opinions and comments from a more theoretical perspective. We are confident that both viewpoints will contribute to assess and improve our composition paradigm and our insights about spatially-aware cross-device interactions in general.

Acknowledgments. This work is partially supported by the Italian Ministry of University and Research (MIUR) under grant PON 02_00563_3470993 "VINCENTE" and by the Italian Ministry of Economic Development (MISE) under grant PON Industria 2015 MI01_00294 "LOGIN".

\section{References}

1. Ardito C., Bottoni P., Costabile M. F., Desolda G., Matera M., and Picozzi M. (2014). Creation and use of service-based Distributed Interactive Workspaces. In Journal of Visual Languages \& Computing. 25, 6, 717-26.

2. Rädle R., Jetter H.-C., Marquardt N., Reiterer H., and Rogers Y. (2014). HuddleLamp: Spatially-Aware Mobile Displays for Ad-hoc Around-the-Table Collaboration. In Proceedings of the Ninth ACM International Conference on Interactive Tabletops and Surfaces (ITS '14), Dresden, Germany.

3. Ardito C., Costabile M. F., and Jetter H.-C. (2014). Gestures that people can understand and use. In Journal of Visual Languages \& Computing. 25, 5, 572-6. 